\title{
Influencia de los Contaminantes Atmosféricos en las Infecciones Respiratorias Agudas en Mexicali-Baja California, México
}

Martha Ramírez-Rembao*, Rosa I. Rojas y Rafael García-Cueto

Universidad Autónoma de Baja California, Instituto de Ingeniería, Av. Obregón 927, Segunda

Sección, Mexicali, B. C.-México (e-mail: excorg@hotmail.com)

*autor a quien debe ser dirigida la correspondencia

\begin{abstract}
Resumen
El objetivo de este estudio es identificar la relación entre los contaminantes: ozono, monóxido de carbono, partículas suspendidas $\left(\mathrm{PM}_{10}\right)$, temperatura y humedad con la incidencia de morbilidad por Infecciones Respiratorias Agudas (IRAs) en el área urbana de Mexicali en el estado de Baja California en México. Se recolectó información de estaciones de monitoreo del aire establecidas en Mexicali, en el período 2001-2005, y se utilizaron modelos de regresión lineal simple y múltiple para analizar estas variables, relacionándolas con IRAs. Se encontró un alto coeficiente de determinación de monóxido de carbono con IRAs en el occidente y centro de la ciudad, bajo con ozono y $\mathrm{PM}_{10} \mathrm{y}$ elevado con temperatura. Basado en los resultados este estudio proporciona, se evidencia la alta relación entre el monóxido de carbono y la temperatura con las infecciones respiratorias agudas.
\end{abstract}

\section{Influence of Atmospheric Pollutants on Acute Respiratory Infections in Mexicali-Baja California, Mexico}

\begin{abstract}
The objective of this research is to identify the relationship between pollutants (ozone, carbon monoxide, $\mathrm{PM}_{10}$ ), temperature and humidity and the morbidity of acute respiratory infections (ARI) in Mexicali, state of Baja California in Mexico. Information was obtained from air monitoring stations in Mexicali, during the period 2001-2005. Simple lineal and multiple regression models were used to relate the variables and acute respiratory infections. A high coefficient of determination of carbon monoxide and ARI was found in the western and central areas of the city. A lower coefficient was found with ozone and suspended particles but temperature showed a high coefficient. Based on the results, this research study provides evidence of the high relationship between carbon monoxide, temperature and ARI.
\end{abstract}

Keywords: air pollutants, Acute Respiratory Infections, ozone, carbon monoxide, suspended particles 


\section{INTRODUCCIÓN}

Actualmente a nivel mundial se presenta una gran preocupación por la contaminación ambiental y la forma en que está afectando al planeta y la salud de la población en general (Smith y Akbar, 2003). Omran (1971), creó el término transición epidemiológica con la que se abrió paso a una verdadera reconceptualización sobre la dinámica de las causas de enfermedad y muerte de la población, proceso dinámico en el cual los patrones de salud y enfermedad de una sociedad se transforman en respuesta a cambios de índole demográfico, económico, tecnológico, político, cultural y biológico.

Las enfermedades por contaminantes ambientales, sobre todo las del aire, son un conjunto heterogéneo de interacciones entre agente y huésped. Considerando el agente debe tomarse en cuenta su toxicidad y concentración, sobre el huésped existen factores como la hipersensibilidad, alteraciones inmunitarias, alteraciones con causas genéticas y psicológicas. Estas pueden presentarse como enfermedades respiratorias agudas o crónicas al estar expuesto a algún contaminante, entre otras como lo menciona Ocaña et al. (2001).

Al referirse a contaminación del aire, las Infecciones Respiratorias Agudas (IRA's) son un problema importante de salud pública y representan una de las principales causas de atención médica a nivel mundial. Son problemas clínicos de etiología múltiple que se presentan en forma aguda o crónica, con variación estacional predominantemente en invierno. La sintomatología se puede localizar en vías respiratorias altas (VRA) en forma aislada o como parte de una enfermedad sistémica con ataque al estado general afectando vías respiratorias bajas (VRB) y parénquima pulmonar (Kasper et al., 2006). Se entiende por IRAs una enfermedad causada principalmente por virus y en segundo lugar por bacterias, que puede presentarse como: catarro común, gripe, rinofaringitis, faringo amigdalitis media, sinusitis, bronquitis, bronconeumonía y neumonía (Rodríguez y Sánchez, 2000).

Las estadísticas disponibles (Batista y Feal, 1988) muestran que entre el 30 y $60 \%$ de las consultas de niños enfermos son por esta causa y un 30 o $40 \%$ de ellos son hospitalizados. Como promedio cada niño presenta de 4 a 8 episodios de IRA's al año. Otro grupo altamente vulnerable son las personas mayores de 60 años. Según estimaciones de la Organización Panamericana de la Salud (OPS) la mortalidad por IRA's en menores de 5 años (incluye influenza, neumonía, bronquitis y bronquiolitis) va desde 16 muertes por cada 100,000 habitantes en Canadá a más de 3,000 en Haití, donde estas afecciones aportan entre 20 y $25 \%$ del total de defunciones en esa edad.

La estadística de la Secretaria de Salud en México, reportó las siguientes tasas de mortalidad por IRAs en la población en general: en el año 2000 una tasa de 14.3 por cada 100,000 habitantes, en 2003 una tasa de 10.7 y en el 2005 una tasa de 14.1. Entre los factores que determinan esta morbilidad por IRAs menciona Kasper et al. (2006), son el bajo peso al nacer, la malnutrición, la contaminación atmosférica, las inadecuadas condiciones de atención médica y de salud. En la actualidad se han hecho estudios en diferentes países: Pope (2004) de Estados Unidos y RosalesCastillo et al. (2001) de México, proveen evidencias de la contaminación del aire y sus efectos adversos en la salud; así como Barrios et al. (2004) de Chile, Ballester et al. (2003) de España y Sinclair y Tolsma (2004) de Estados Unidos, quienes concluyen que la contaminación atmosférica es uno de los principales problemas que afectan la salud de la población.

Mexicali cuenta con diferentes actividades productivas que contribuyen a que esta ciudad sea considerada la tercera ciudad mas contaminada del país (Reyna et al., 2003). Una de las actividades que incrementa los niveles de contaminación del aire según Moncada (2006), son las quemas de residuos agrícolas del Valle de Mexicali, con lo que se generó en 2003 13,870,490 kg de monóxido de carbono y en $200420,181,295 \mathrm{~kg}$, siendo este uno de los contaminante que mostró mayores cifras en estas quemas. Otra actividad productiva es la industria, que según los estudios de Nieblas (2006), también son responsables de la acumulación de contaminantes, generando en la ciudad de Mexicali en 2003 2,281 tons. de monóxido de carbono (CO), 2,094 tons. de óxido de nitrógeno (NOx) y 1,094 tons. de partículas suspendidas $\left(\mathrm{PM}_{10}\right)$, afectando la salud de la población.

Otros estudios en diferentes países han encontrando relación de la incidencia de IRA's con diferentes factores del medio, como Romero-Placeres et al. (2004) y Molina et al. (2001) en sus 
estudios en La Habana, Cuba, mencionan una elevada asociación entre contaminantes del aire como $\mathrm{PM}_{10}$ y los casos de IRA's. Collins et al. (2003) mencionan también en sus estudios, a los materiales en forma de partículas que permanecen suspendidos en la atmósfera por periodos de tiempo prolongados a una altura que las personas pueden respirar y a partículas mas finas que tienen la capacidad de penetrar a los tejidos internos del tracto respiratorio y producir potencialmente complicaciones de salud muy severas. La Agencia de Protección Ambiental de Estados Unidos (EPA, 2005) ha definido nuevos estándares para las partículas menores a 2.5, clasificándolas como componentes tóxicos del aire, capaces de penetrar a las vías aéreas y alvéolos pulmonares (Samet et al. 2000).

Se identifica la relación con otros contaminantes con los trabajos de Brunekeef et al. (2000) quien establece en su estudio en los Países Bajos la relación entre mortalidad y los contaminantes $\mathrm{PM}_{10}$ y $\mathrm{O}_{3}$, mientras que Hajat et al. (2002), encontraron en su estudio en Londres asociación entre el contaminante $\mathrm{PM}_{10}, \mathrm{O}_{3}$ y $\mathrm{SO}_{2}$ y la incidencia de IRA's en adultos mayores y en niños, en los meses fríos. Andrade et al. (2006), en México en un estudio de correlación, encontraron relación significativa con monóxido de carbono y otros contaminantes, mas no con ozono y $\mathrm{PM}_{10}$.

Hernández-Cadena et al. (2007), en su estudio también en México, en el que utilizó un modelo aditivo generalizado, asumiendo una distribución Poisson en donde las concentraciones de ozono pero no las de $\mathrm{PM}_{10}$ tienen relación significativa con Infecciones Respiratorias Agudas en niños de 5 años y menores.

Complementando lo anterior Ramírez-Sánchez et al. (2006), en su estudio ecológico en México señala que los contaminantes atmosféricos $\mathrm{CO}$ y $\mathrm{NO}_{2}$ muestran correlación significativa con las IRA's en niños menores de 5 años en el área urbana de la ciudad de Guadalajara, México. El monóxido de carbono incide en las infecciones respiratorias agudas, pese a que las concentraciones se mantienen por debajo de la norma. Caso semejante el reportado por Morales (2003) y ArribasMonzon et al. (2001) quienes consideran que aunque parte del día las concentraciones de contaminantes son bajas o no superan los límites establecidos por las normas, si muestran un efecto negativo en la salud.

Como se aprecia en los estudios realizados en diferentes países, todo ser humano en respuesta a ciertos estímulos en ocasiones persistentes en su entorno presentan signos y síntomas, ocasionando inflamación en el tracto respiratorio, que al presentarse en un individuo comprometido inmunológicamente ocasiona mayor labilidad a agentes infecciosos como virus, bacterias, hongos, etc. provocando una infección respiratoria aguda tendiendo a verse severamente afectados. Boezen et al. (2005), en sus estudios en Amsterdam concluyó que la hiperreactividad de vías respiratorias y la inmunoglobulina IGE alta, son características de la población adulta que los hace más susceptibles de respuesta ante contaminantes del aire.

El objetivo del presente estudio es encontrar la relación existente entre los contaminantes: ozono, monóxido de carbono y partículas suspendidas, en este caso las menores de 10 micrómetros de diámetro $\left(\mathrm{PM}_{10}\right)$ con la incidencia de IRAs en la población del área urbana de Mexicali. B. C. en los años 2001 a 2005. Contaminantes sobre los que se obtiene información de las 3 Estaciones de Monitoreo, aunque no completa como sería deseable. Se incluyen también factores climáticos como temperatura y humedad por considerarse de importancia al analizar el ambiente y la salud del ser humano.

Los resultados aportarán información para reforzar programas de medicina preventiva dirigidos al control de algunos elementos que contribuyen a la incidencia de IRAs y lograr con esto una disminución en las tasas de morbilidad y mortalidad.

El proceso de desarrollo de la ciudad de Mexicali desde su origen en 1903 hasta el 2007, ha manifestado en sus diferentes etapas una dinámica que ha creado el problema de contaminación del aire, asociado con sus principales actividades productivas. Como la actividad agrícola, con la quema de cultivos y el uso de sustancias químicas, el incremento de la industria que en 2005 contaba con 1094 establecimientos en la ciudad según la Secretaria de Desarrollo Económico (SEDECO, 2005), 
también por el incremento de población y aumento del parque vehicular, que han elevado la generación de contaminación del aire por la quema de combustibles fósiles.

De esta forma se tiene ahora una ciudad con 878,194 habitantes (INEGI, 2005) y problemas de contaminación del aire que la ubican entre las 5 ciudades que rebasan las normas al año para $\mathrm{PM}_{10}$, $\mathrm{NO}_{2}, \mathrm{SO}_{2}, \mathrm{CO}$ y $\mathrm{O}_{3}$ (INEGI, 2007) y por el lado de salud a nivel nacional, Baja California ocupa el $19^{\circ}$ lugar en morbilidad por IRAs (SS, 2005).

\section{MATERIAL Y MÉTODOS}

El diseño del presente estudio se llevó a cabo mediante las siguientes etapas:

1. Sobre población, la tasa de crecimiento anual para el periodo 2001-2005 se obtuvo con base en las cifras del XII Censo de Población y Vivienda del Instituto Nacional de Estadística, Geografía e Informática 2000 (INEGI, 2000) y proyecciones de población por el Consejo Estatal de Población de Baja California (CONEPO, 2005), para contar con la información de población de las 350 colonias del área urbana de la ciudad de Mexicali. La ciudad cuenta con 13 Centros de Salud que prestan atención médica de primer nivel al 32\% de la población total, que es la población abierta o población no derechohabiente de Servicios de Seguridad Social, distribuyendo la Secretaria de Salud las 350 colonias en los 13 centros de salud (figura 1).

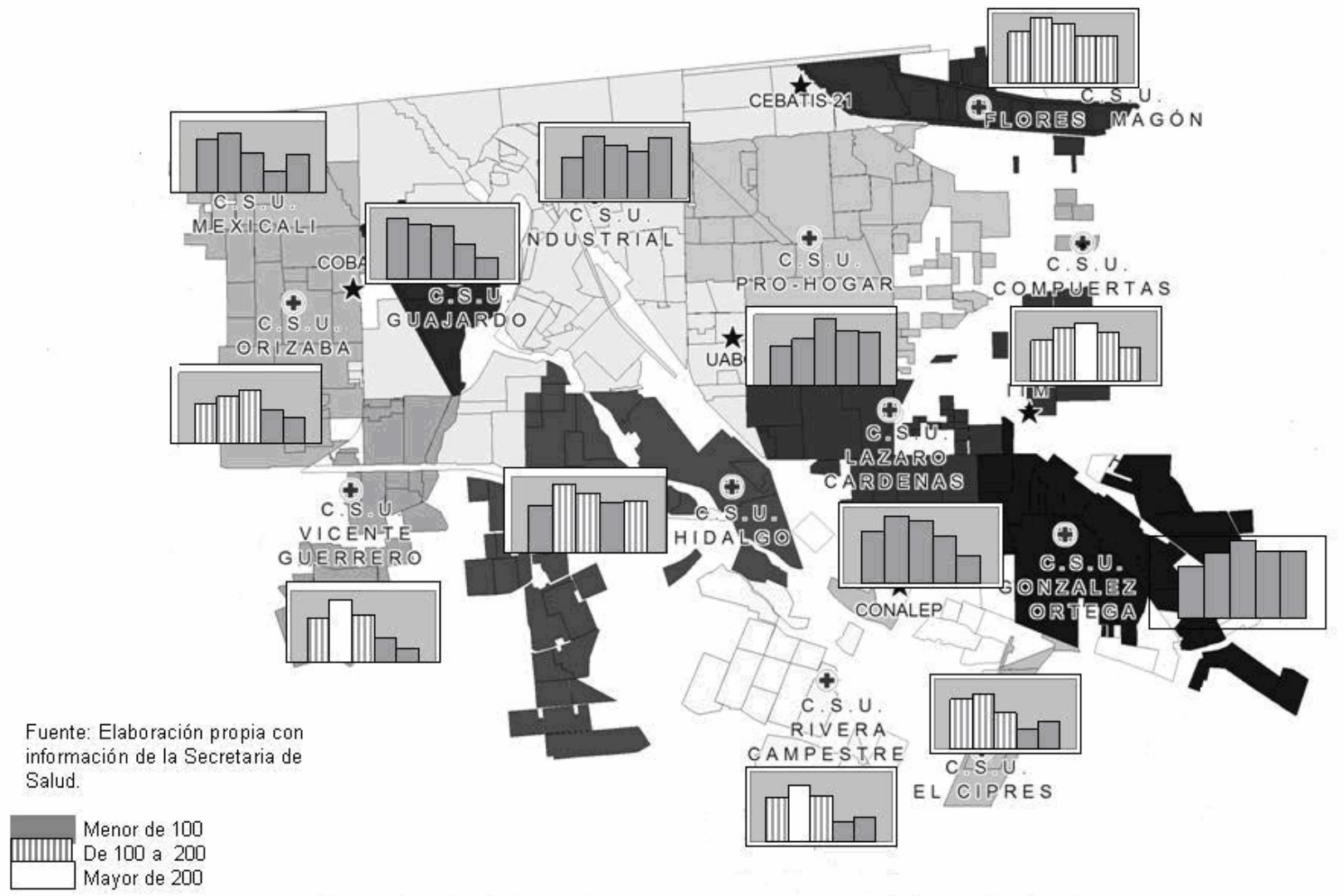

Fig. 1: Tasas de IRAs de los 13 Centros de Salud de Mexicali, B. C. 2001-2005

2. Se registró el total de IRAs de primera vez de cada Centro de Salud de la población abierta. De la información recabada se obtienen indicadores de morbilidad, esto es la tasa de IRA's por 1,000 habitantes (San Martín, 1988) en cada uno de los 13 centros de salud urbanos de 2001 a 2005, mostrados en la Figura 1.

3. Sobre los contaminantes del aire, se recabó información en la ciudad de Mexicali de ozono $\left(\mathrm{O}_{3}\right)$ y monóxido de carbono (CO) de los años 2001 a 2005 en 3 Estaciones de Monitoreo: UABC, ITM y COBACH. De $\mathrm{PM}_{10}$ se capturó información en 5 estaciones de monitoreo UABC, ITM, COBACH, PROGRESO y CONALEP de 2001 a 2003. Es importante hacer notar que estas estaciones de monitoreo no cubrieron en forma constante, el total del área urbana de la ciudad. (figura 2) 


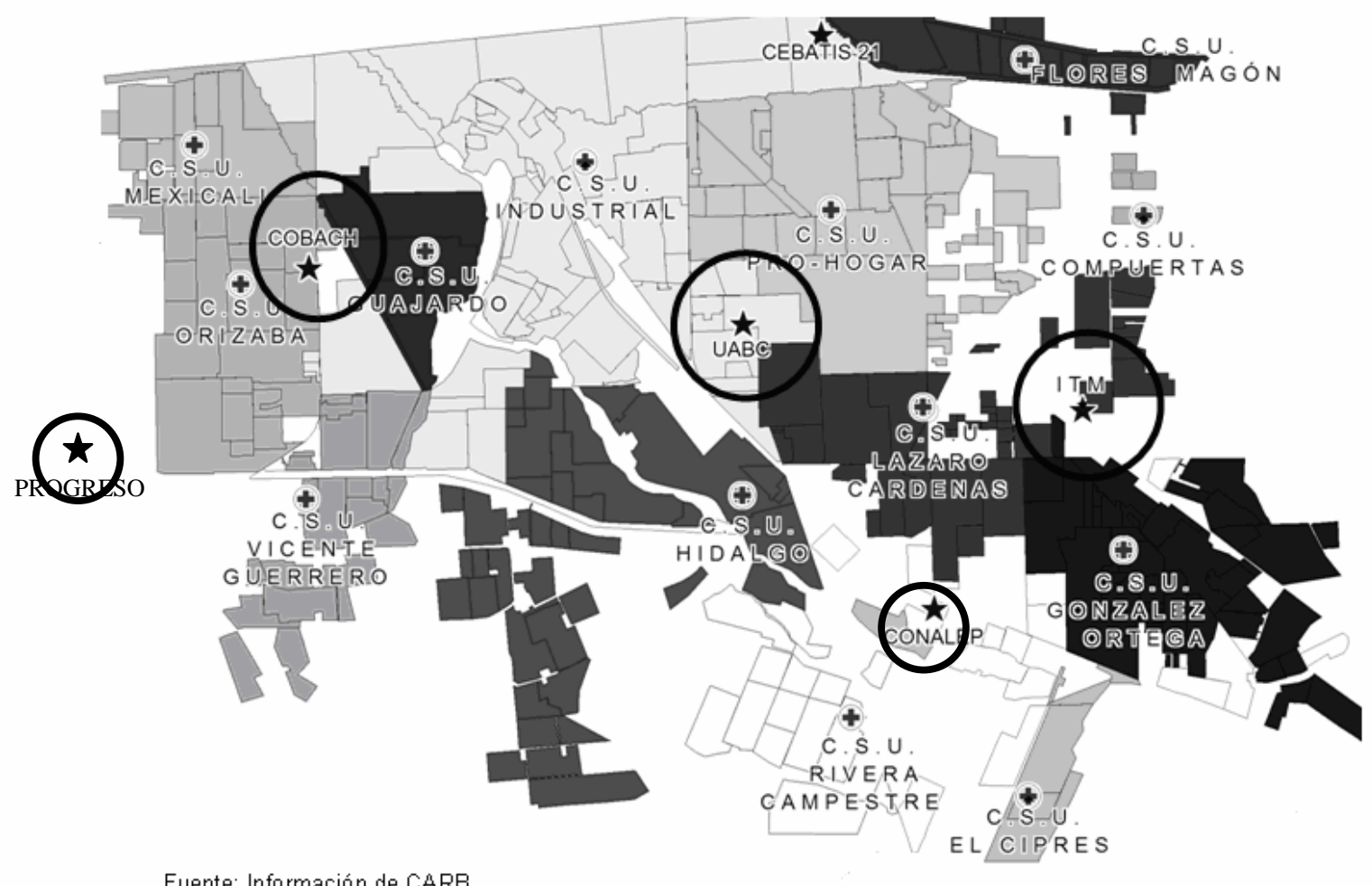

Fuente: Información de CARB.

Fig. 2: Ubicación de Estaciones de Monitoreo en Mexicali, B. C. 2001-2005

En las estaciones de monitoreo la información se recaba de la siguiente manera:

Para las partículas como el $\mathrm{PM}_{10}$ se utiliza un muestreador de alto volumen que captura este contaminante, Wedding Beta Gauge Sampler. Para gases como el $\mathrm{O}_{3}$ se utilizan equipos como el DASIBI 1003 (analizador fotométrico de ozono UV con generador) y el API 400 (advanced polution incorporation), y para el CO se utilizan equipos como el DASIBI 3008 (Gas filter correlation Co. analyzer), TECO 48 y ASPI 300, diferentes en cada estación de monitoreo según equipo disponible. En cada una de las estaciones se captura información cada segundo, concentrándola en un promedio de 10 segundos, posteriormente se toma un promedio parcial de 6 tomas o 60 segundos o un minuto, así se continúa con los promedios hasta tener el valor que se registra, que es el promedio horario mensual.

La UABC y otras instituciones de educación en Mexicali proporcionan los espacios para la instalación de los equipos de monitoreo. TRASER contratista de San Marcos, Ca. USA es la empresa responsable de estas estaciones y es quien recaba la información, para posteriormente enviarla a Air Quality System AQS (EPA) en donde se revisa y una vez validada por ellos, se notifica a TRASER para que se suba a la red. Para determinar la concentración de una sustancia química en un volumen, se utilizan las ppb (partes por billón) utilizadas para determinar las concentraciones muy pequeñas de gases en la atmósfera. La información de contaminantes recibida para su análisis fue enviada para el presente estudio directamente de los registros de Air Quality System AQS (EPA) por medios electrónicos. En el presente estudio se seleccionan los valores máximos mensuales registrados de cada estación de monitoreo, por considerarse que son los que pudieran dañar la salud. Se localizan las estaciones de monitoreo (figura 2) considerando los centros de salud que se encuentra más próximos, para conocer la relación de la concentración de contaminantes con la morbilidad por IRAs en esa área. Como parámetros para los contaminantes se emplean las Normas Oficiales Mexicanas, para ozono la NOM-020-SSA1 (1993), para monóxido de carbono la NOM-021SSA1 (1993) y para partículas suspendidas PM10 y PM 2.5 la NOM-025-SSA1 (1993). 
4.- Se incluyen en el análisis las variables climáticas temperatura y humedad relativa del aire que se obtuvo del Reporte Meteorológico 2001-2005 del Departamento de Meteorología del Instituto de Ingeniería de la Universidad Autónoma de Baja California, que mide la temperatura en grados centígrados y la humedad relativa (HR) que se expresa en porcentaje. Se seleccionan temperaturas mínimas promedio mensuales por considerarse lo que mayormente afectan la salud y en el caso de la humedad los promedios máximos mensuales.

Sobre el comportamiento del viento, el Programa para mejorar la calidad del aire en Mexicali 20002005 encontró que en Mexicali el flujo del viento a lo largo del año se diferencia en dos patrones: los vientos de componente occidental se presentan con una frecuencia de $69 \%$ y ocurren principalmente en los meses de octubre a junio. El patrón de vientos orientales con una frecuencia de $31 \%$ ocurren principalmente en los meses de julio a septiembre. Este comportamiento estacional es importante para el transporte de material particulado y de contaminantes de un lado de la frontera al otro, sin embargo este patrón de vientos no necesariamente se refleja en la marcha anual de los contaminantes, ya que hay otras variables implicadas, como puede ser la radiación solar, temperatura del aire ó la estabilidad atmosférica que es causada por el anticiclón casi-permanente que domina en la región y que da lugar al clima semidesértico regional.

La red de Monitoreo de la calidad del aire en Mexicali muestra información obtenida en las estaciones de monitoreo COBACH, ITM y UABC, en las que se observa lo siguiente:

-El comportamiento del viento en las estaciones de monitoreo es muy parecido, destacando que en todas ellas el viento mas frecuente proviene tanto del sureste como del noroeste.

-De manera general se puede destacar también que la frecuencia promedio del viento del sureste es aproximadamente $14 \%$, en tanto que la frecuencia de ocurrencia de los vientos del noroeste es aproximadamente $12 \%$.

En cuanto a la velocidad del viento se apreció que la intensidad mas frecuente, en todas las estaciones de monitoreo, se ubica por debajo de $2 \mathrm{~m} / \mathrm{s}$.

Sobre lluvia, el mismo Programa menciona que el terreno plano del valle y las fuertes gradientes de temperatura creada por el intenso calentamiento solar producen vientos moderados y una profunda convección térmica. La combinación de subsidencias con el efecto protector de las montañas y la distancia con respecto al océano limita severamente la precipitación, la cual es altamente variable y en ocasiones una fuerte precipitación puede constituir casi el total anual. Debido a la baja cantidad de lluvia, solamente $75 \mathrm{~mm}$ al año, el lavado atmosférico es infrecuente por lo que esta variable no se consideró importante para el estudio realizado.

Se elaboró una base de datos mensual de los años 2001 a 2005 de los contaminantes: ozono, monóxido de carbono y de 2001 a 2003 del $\mathrm{PM}_{10}$, y las condiciones atmosféricas temperatura y humedad de cada Estación de Monitoreo, comparándolo con las tasas de IRAs de los Centros de Salud Urbanos. Esta información se procesó con el Programa STATISTICA 7 con el que se realizó un análisis de Regresión Lineal, simple y múltiple, para estudiar la tasa de morbilidad por IRAS en cada centro de salud, como variable respuesta, y contaminantes y variables atmosféricas, como variables predictoras. Esta modelación tiene el propósito de identificar cuales variables explican mejor el problema de la presencia de las IRAs por centro de salud estudiado.

\section{RESULTADOS Y DISCUSIÓN}

Tomando como base lo señalado por las Normas Oficiales Mexicanas, como ejemplo se muestra en la figura 3 el monóxido de carbono registrado por las 3 estaciones de monitoreo que rebasa los limites de la norma de 43 a 51\% de los meses en los 5 años. El ozono rebasa el límite establecido por la norma $31 \%$ de los meses, solo en la estación de monitoreo UABC. EI PM 10 rebasa los limites el $72 \%$ de los meses en la estación de monitoreo $\mathrm{COBACH}$.

Lo anterior muestra que en el área monitoreada en la ciudad de Mexicali, los contaminantes que superan los límites aceptables para la protección de la salud según las Normas Oficiales Mexicanas 
se presentan en diferentes zonas y en diferentes proporciones, teniendo en primer lugar al monóxido de carbono, seguido por el $\mathrm{PM}_{10}$ y posteriormente el ozono.

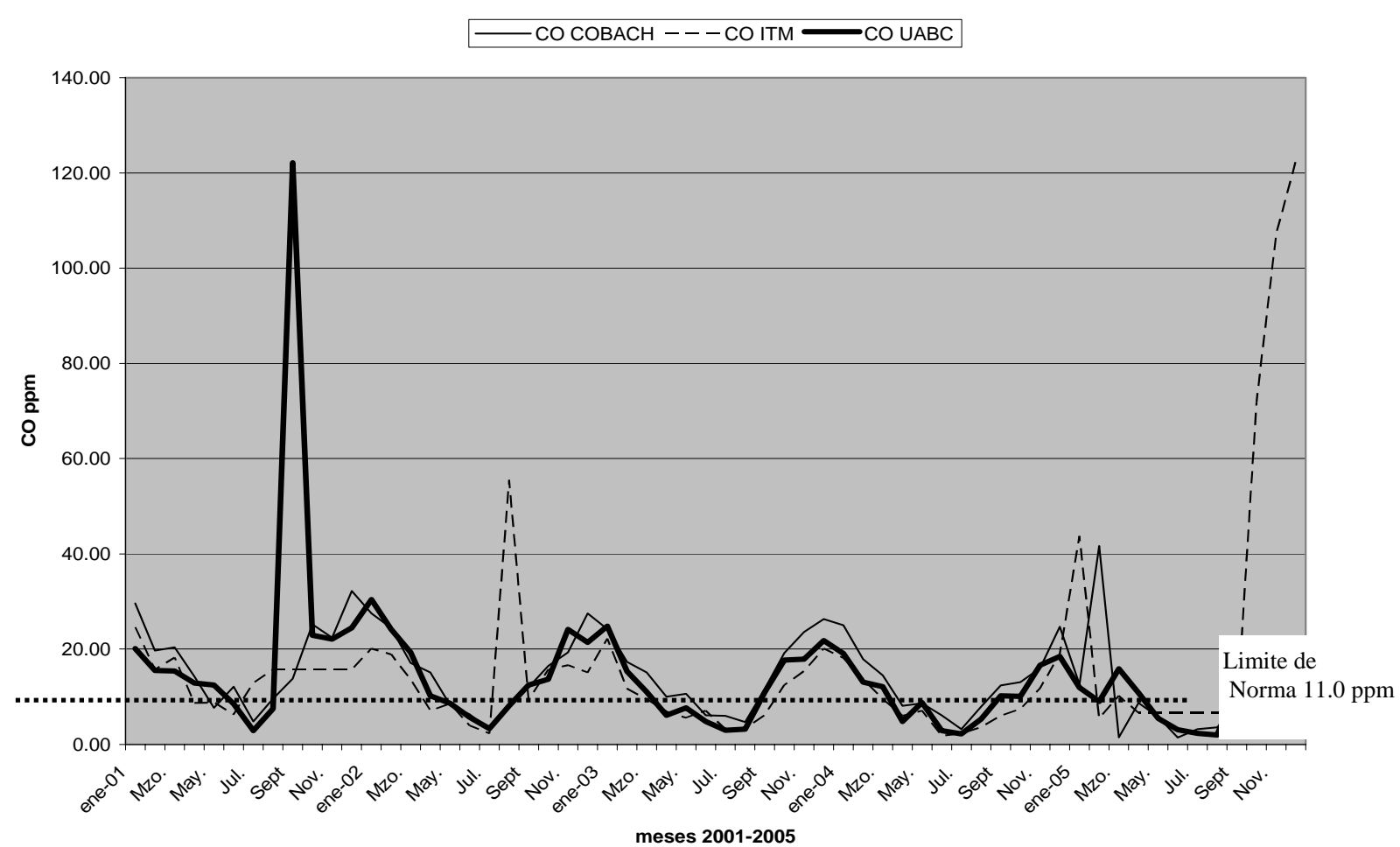

Fig. 3: El monóxido de carbono rebasa el límite de la norma en las 3 estaciones de monitoreo.

El análisis estadístico de la información recabada en la ciudad de Mexicali de 2001 a 2005, señala el coeficiente de determinación de las IRA's con el contaminante Ozono, Monóxido de Carbono y de $\mathrm{PM}_{10}$ de 2001 a 2003 y las condiciones atmosféricas temperatura y humedad.

En la tabla 1 se presentan los coeficientes de determinación obtenidos al realizar el análisis de regresión lineal simple con las IRAs como variable dependiente y los contaminantes y variables meteorológicas como variables independientes. Las variables que mayormente explican a las IRAs son por orden de importancia temperatura, monóxido de carbono y ozono, mientras que el $\mathrm{PM}_{10}$ y humedad relativa no son significativos.

Tabla 1: Coeficientes de determinación de IRAs y contaminantes $\mathrm{O}_{3}, \mathrm{CO}$ y $\mathrm{PM}_{10}$, temperatura y humedad, Mexicali, B. C.

\begin{tabular}{|l|c|c|c|c|c|}
\hline $\begin{array}{c}\text { CENTROS DE } \\
\text { SALUD }\end{array}$ & $\begin{array}{c}\mathbf{O}_{\mathbf{3}} \\
\mathbf{2 0 0 1 - 2 0 0 5}\end{array}$ & $\begin{array}{c}\mathbf{C O} \\
\mathbf{2 0 0 1 - 2 0 0 5}\end{array}$ & $\begin{array}{c}\mathbf{P M}_{\mathbf{1 0}} \\
\mathbf{2 0 0 1 - 2 0 0 3}\end{array}$ & $\begin{array}{c}\text { TEMPERATURA } \\
\mathbf{2 0 0 1 - 2 0 0 5}\end{array}$ & $\begin{array}{c}\text { HUMEDAD } \\
\mathbf{2 0 0 1 - 2 0 0 5}\end{array}$ \\
\hline Industrial & 0.24 & 0.19 & 0.003 & 0.57 & 0.01 \\
\hline Pro-Hogar & 0.36 & 0.15 & 0.0006 & 0.51 & 0.05 \\
\hline Hidalgo & 0.27 & 0.34 & 0.02 & 0.51 & 0.01 \\
\hline Glez.Ortega & 0.03 & 0.01 & 0.07 & 0.57 & 0.04 \\
\hline Compuertas & 0.01 & 0.04 & 0.01 & 0.39 & 0.04 \\
\hline L.Cardenas & 0.003 & 0.02 & 0.08 & 0.28 & 0.01 \\
\hline Guajardo & 0.07 & 0.47 & 0.13 & 0.43 & 0.07 \\
\hline Mexicali & 0.005 & 0.19 & 0.04 & 0.29 & 0.01 \\
\hline Orizaba & 0.023 & 0.42 & 0.01 & 0.51 & 0.04 \\
\hline V.Guerrero & 0.011 & 0.27 & 0.05 & 0.26 & 0.003 \\
\hline Campestre & - & & & 0.35 & 0.009 \\
\hline El Cipres & - & & & 0.27 & 0.003 \\
\hline Flores Magon & - & & & 0.22 & 0.005 \\
\hline
\end{tabular}


Del análisis de la Tabla 1, se tiene la siguiente información:

a) En relación al ozono solo en el centro de la ciudad, se presenta un coeficiente de determinación alto con las IRAs en el Centro de Salud Pro-hogar y más bajo en los otros dos Hidalgo e Independencia los tres de la Estación de Monitoreo UABC.

b) En el caso del Monóxido de Carbono, al oeste de la ciudad este contaminante muestra un coeficiente de determinación alto con las IRAs en el Centro de Salud Guajardo y Orizaba, mas bajo en los dos restantes de la Estación de Monitoreo COBACH. También en el centro de la ciudad se presenta coeficiente de determinación alto en el centro de salud Hidalgo y más bajo en los otros dos centros de salud.

c) Sobre $\mathrm{PM}_{10}$ de la información disponible de 2001 a 2003, se obtiene un coeficiente de determinación bajo en este periodo de tiempo, en todos los centros de salud.

Resumiendo, se observa que el $\mathrm{O}_{3}$ solo en tres centros de salud de la estación de monitoreo UABC en el centro de la ciudad explica casi $1 / 3$ parte del comportamiento de las IRAs.

En el caso del monóxido de carbono en la estación de monitoreo $\mathrm{COBACH}$ al oeste de la ciudad tienen un poder explicativo considerable en dos centros de salud casi con el $50 \%$ de las IRAs, y en los otros centros de salud de esta área mas bajos. En el centro de la ciudad se explica en un centro de salud casi una 1/3 parte y mas bajo en los otros dos centros de salud.

d) A diferencia de lo anterior, todos los centros de salud muestran una relación alta de las IRA's con la temperatura.

Se consideró importante analizar con regresión múltiple la información de estos contaminantes tomando en cuenta la variable temperatura, que mostró mayor significancia en forma individual, con mayor relación estadística (tabla 1). Se puede ver con este análisis de regresión múltiple, que en términos relativos las variables $\mathrm{PM}_{10}$ y temperatura, explican en mayor proporción las IRAs.

En el estudio de Reyna et al. (2003), en la ciudad de Mexicali, el detecta en un análisis de regresión múltiple un coeficiente de determinación de 0.83 de IRAs con temperatura y $\mathrm{PM}_{10}$ de 1997 a 2000 y en el presente estudio de 2001 a 2005 con ambas variables se obtuvo un coeficiente de determinación de 0.42 a 0.68 en diferentes áreas de Mexicali (tabla 2). Los estudios de HernándezCadena et al. (2007) y Ramírez-Sánchez et al. (2006), no se pueden comparar con el resultado del presente estudio, pues ellos trabajaron con un sector de la población de 5 años y menores.

Tabla 2: Coeficientes de determinación de IRAs con contaminantes y temperatura, Mexicali

\begin{tabular}{|l|c|c|c|}
\hline \multicolumn{1}{|c|}{$\begin{array}{c}\text { CENTROS DE } \\
\text { SALUD }\end{array}$} & $\begin{array}{c}\mathbf{O}_{\mathbf{3}} \text { y temp } \\
\mathbf{2 0 0 1 - 2 0 0 5}\end{array}$ & $\begin{array}{c}\text { CO y temp. } \\
\mathbf{2 0 0 1 - 2 0 0 5}\end{array}$ & $\begin{array}{c}\text { PM }_{\mathbf{1 0}} \text { y temp. } \\
\mathbf{2 0 0 1 - 2 0 0 3}\end{array}$ \\
\hline Industrial & 0.58 & 0.57 & 0.62 \\
\hline Pro-Hogar & 0.58 & 0.51 & 0.51 \\
\hline Hidalgo & 0.55 & 0.58 & 0.62 \\
\hline Glez.Ortega & 0.57 & 0.58 & 0.55 \\
\hline Compuertas & 0.39 & 0.39 & 0.44 \\
\hline L.Cardenas & 0.28 & 0.29 & 0.62 \\
\hline Guajardo & 0.45 & 0.52 & 0.61 \\
\hline Mexicali & 0.35 & 0.30 & 0.68 \\
\hline Orizaba & 0.52 & 0.53 & 0.61 \\
\hline V.Guerrero & 0.28 & 0.30 & 0.42 \\
\hline Campestre & - & - & - \\
\hline El Cipres & - & - & - \\
\hline Flores Magon & - & - & \\
\hline
\end{tabular}

Se muestra como ejemplo la ecuación del centro de salud Hidalgo, por ser uno que presenta coeficiente de determinación más alto, aplicable únicamente para las cercanías de este centro de salud.

La ecuación que explica la cantidad de IRA's en el centro de salud Hidalgo, de la estación de monitoreo $\mathrm{UABC}$, con un coeficiente de determinación alto de 0.68, con los 3 contaminantes: $\mathrm{O}_{3}$, 
$\mathrm{CO}, \mathrm{PM}_{10}$ y temperatura y humedad de 2001 a 2003 en la ciudad de Mexicali, se muestra a continuación:

IRAs C.S. Hidalgo $=30.5-70.2^{*} \mathrm{O}_{3}(\mathrm{UABC})+0.03^{*} \mathrm{CO}(\mathrm{UABC})+0.0001^{*} \mathrm{PM} 10(\mathrm{UABC})-0.42 * \operatorname{temp}-0.11 * \mathrm{HR}$

El resultado del presente estudio arrojó cifras que muestran que el monóxido de carbono (CO) contribuyó con cifras mayores a la incidencia de morbilidad por IRAs en la ciudad de Mexicali en el oeste y centro de la ciudad de 2001 a 2005, en las estaciones de monitoreo COBACH y UABC, pues en un análisis de regresión lineal simple este contaminante mostró coeficientes de determinación más altos que el ozono y el $\mathrm{PM}_{10}$, en un territorio más extenso.

Esto de acuerdo con lo que concluye en su estudio Ramírez-Sánchez et al. (2006), quién con un análisis de correlación encontró que el monóxido de carbono (CO) incide en la salud de la población infantil del área urbana de Guadalajara, Jalisco, México, de 2000 a 2002, indicando que la población esta en contacto con exposiciones fuera de norma algunas horas de ciertos días al mes, lo que influyó de manera significativa en el incremento de las IRAs.

Esto en la ciudad de Mexicali podría deberse a la cantidad de vehículos en circulación que ha aumentado considerablemente en estos últimos 6 años, pues según el H. Ayuntamiento de Mexicali (2006), de 181,300 unidades 1 por cada 3 habitantes en 1998, se incrementó a 320,000 en 2004, esto es 1 por cada 2 habitantes, un incremento de un 57\% en este período de 6 años. Considerando que según el Programa de Mejora de la Calidad del Aire en Mexicali 2000-2005, en 1996 el 95\% de la flota vehicular en Mexicali eran modelos 1990 y anteriores.

También según los AFOROS registrados por el $\mathrm{H}$. Ayuntamiento de Mexicali, que informan que en 2004 existieron vías y cruceros en donde transitaron en un día más de 56,000 automóviles y otras vías o cruceros con cantidades similares. Sumado a esto, la Garita Internacional México-Estados Unidos reportó que en el año 1996 cruzaron la línea fronteriza un total de 6,989,639 vehículos, unidades que permanecen haciendo fila generalmente mas de 1 hora, y al aumentar anualmente la población y el parque vehicular, esta cifra seguramente se incrementó, con una alta emisión de contaminantes por la cantidad de horas motor al año. Otras actividades productivas de la ciudad serían las mencionadas por Moncada (2006) con las quemas de residuos agrícolas del Valle de Mexicali y las industrias generadoras de electricidad mencionadas por Nieblas (2006).

En relación al ozono, el resultado del análisis de regresión mostró cifras bajas al relacionarlo con las IRAs, solo en la estación de monitoreo UABC del centro de la ciudad las cifras son altas, pero negativas con una relación inversa, contrario con lo mencionado por Hajat et al. (2002) que encontraron en sus estudios alta relación del ozono con las IRAs. Así como con el resultado de Hernández-Cadena et al. (2007), quien utilizando modelo aditivo generalizado, asumiendo una distribución de Poisson, menciona como resultado que las concentraciones de ozono se asocian significativamente con IRAs, pero no las de $\mathrm{PM}_{10}$ en niños de 5 años y menores.

En relación al $\mathrm{PM}_{10}$, se cuenta con los resultados del estudio hecho por Reyna et al. (2003) de Mexicali, México, quién utilizando coeficientes de correlación y regresión de Poisson, encuentra relación del $\mathrm{PM}_{10}$ con las IRAs. Diferente al resultado en este estudio, con información también en la ciudad de Mexicali pero de 2001 a 2003, en el que con análisis de regresión se obtuvieron coeficientes de determinación bajos, indicando que se tiene poca relación del $\mathrm{PM}_{10}$ con las IRAs en toda la ciudad, esto apoyado con los resultados de Hernández-Cadena et al. (2007) quien encontró también que no había relación de $\mathrm{PM}_{10}$ con IRAs.

Sobre la relación de las condiciones climáticas de temperaturas y humedad relativa con la incidencia de IRAs de 2001 a 2005, se puede mencionar que en Mexicali en el presente estudio resultó en el modelo de regresión lineal simple alta relación con la temperatura, pero baja con la humedad relativa. Esto de acuerdo con Barrios et al. (2004) quien concluye en sus estudios una alta relación de la temperatura ya que las consultas resultaron en mayor número en invierno. Reyna et al. (2003), también menciona en su estudio hecho en la ciudad de Mexicali, como resultado en graficas de 
dispersión y coeficientes de correlación, cifras altas de la temperatura con IRAs, pero baja con la humedad relativa. Resultado de acuerdo con el presente estudio, lo que pudiera deberse a que en esta ciudad de Mexicali el clima es seco.

Para que esta información pueda ser de mayor utilidad en un trabajo de investigación de contaminantes del aire y su relación con la salud, es necesario contar con estadística de salud más completa, de todas las Instituciones de salud públicas y privadas y con estaciones de monitoreo ubicada en puntos estratégicos de toda el área urbana y rural de Mexicali, midiendo mayor cantidad de contaminantes.

\section{CONCLUSIONES}

Se alcanzo el objetivo del presente estudio, ya que se identificó que los contaminantes: ozono, monóxido de carbono, $\mathrm{PM}_{10}$ y los factores climáticos temperatura, humedad, mostraron diferente relación con las infecciones respiratorias agudas (IRAs) en la población de la ciudad de Mexicali, B. C. de 2001 a 2005, como se explica a continuación:

1. El monóxido de carbono y la temperatura ambiente son las variables que mayormente contribuyeron en la incidencia de morbilidad por Infecciones Respiratorias Agudas (IRAs) en la ciudad de Mexicali en el periodo de 2001 a 2005.

2. Contrario a los que algunos autores concluyen en sus estudios sobre la relación del ozono con las IRAs, el resultado del presente estudio manifiesta que sobre este contaminante solo en el centro de la ciudad se encontró evidencia de que contribuyera a la morbilidad por infecciones respiratorias agudas (IRAs) en la ciudad de Mexicali de 2001 a 2005, no en otras áreas.

3. También contrario a los que algunos autores concluyen en sus trabajos, en el presente estudio no se encontró evidencia de que $\mathrm{PM}_{10}$ tenga relación con las IRAs en la ciudad de Mexicali de 2001 a 2003. De igual forma con humedad relativa de 2001 a 2005.

Con la información del presente estudio es necesario identificar las fuentes de generación de contaminantes en las diferentes zonas de la ciudad y con el apoyo de las autoridades, implementar programas para el control de estas fuentes diversas de generación de contaminantes.

\section{REFERENCIAS}

Andrade M., M. Ramírez y M. González; Los contaminantes atmosféricos y su correlación con los casos de infecciones agudas de las vías respiratorias superiores en niños menores de 5 años del área urbana de Guadalajara, Jalisco del 2000 al 2002, Salud Publica de México: 48(5), 385-394 (2006).

Arribas-Monzón F. y otros cinco autores; Efectos de la contaminación atmosférica sobre la mortalidad diaria en la ciudad de Zaragoza, España, 1991-1995, Salud Pública de México: 43(4), 289-297 (2001).

Ballester F. y otros doce autores; Relación a corto plazo de la contaminación atmosférica y la mortalidad en 13 ciudades españolas, Med. Clin. (Barc.):121(18), 684689 (2003).

Barrios C, F. Peña-Cortez y S. Osses; Effects for particles material atmospheric pollution on acute respiratory diseases in under 5 years of age, Ciencia y Enfermería: 10(2), 21-29 (2004).

Batista R. y P. Feal; Las Infecciones Respiratorias Agudas: Un problema siempre Emergente, Unidad Nacional de Análisis y Tendencias en Salud, Ministerio de Salud Publica, 11(2), 63-66 (1988).

Brunekreef H., A. Verhorff, J Wijnen y P Fischer; Daily mortality and air pollution in the Netherland, Journal of Air \& Waste Managment Association: 50(8), 1380-9 (2000). 
Boezen, H. y otros siete autores; Susceptibility to air pollution in elderly males and females, European Respiratory Journal: 25(6), 1018-1024 (2005).

Collins K., M. Quintero, M. Reyna y C. Yruretagoyena; Understanding Air Pollution and Health in the Binational Airshed of the Imperial and Mexicali Valleys, Southwest Center for Environmental Research and Policy, Summer 2003, Revised, 1-10 (2003).

CONEPO, Consejo Estatal de Población del Estado de Baja California; Proyección de población de 2001 a 2005 (2005).

EPA (Environmental Protection Agency); Agencia de Protección ambiental de Estados Unidos, Nuevo estándar de partículas finas (2005).

Hajat S., R. Anderson W. Atkinson y A. Haines; Effects of air pollution on general practitioner consultations for upper respiratory diseases in London, Occupational Environmental Medicine: 59, 294-299 (2002).

Hernández-Cadena L. y otros seis autores; Mortalidad infantil por causas respiratorias y su relacion con la contaminación atmosférica en Cd, Juárez, Chihuahua, México, Salud Publica Mex.: 49, 27-36 (2007).

INEGI; Instituto Nacional de Estadística, Geografía e Informática, XII Censo General de población y Vivienda (2000).

INEGI; Instituto Nacional de Estadística, Geografía e Informática, II Conteo de Población y Vivienda (2005).

INEGI; Instituto Nacional de Estadística, Geografía e Informática, Calidad del Aire (2007).

Kasper D. y otros cinco autores; Principles of Internal Medicine Harrison`s, de Drazen J. et al, Mc Graw-Hill, Health Professions Division, 16 th Edition. New York USA.McGraw-Hill Health Profession Division, IX: 1653-1662 (2006).

Molina E., y otros cuatro autores; Crisis de asma y enfermedades respiratorias agudas. Contaminantes atmosféricos y variables meteorológicas en Centro Habana, Rev. Cubana Med. Gen. Integr.: 17(1), 10-20 (2001).

Moncada A.; Las quemas agrícolas en los valle Imperial de California EUA y Mexicali, B. C. México, En: Contaminación y Medio Ambiente en Baja California, Quintero M. Ed. Porrua, México, 5, 125-135 (2006).

Morales R.; Contaminación del aire y efectos tóxicos por partículas respirables (PM10) en el humo, de madera en comercios de alimentos San José-Costa Rica, Rev. Costarricense de Salud Pública: 12(22), 16-28 (2003).

Nieblas E.; Planeación Ambiental del sector eléctrico en el contexto transfronterizo de California (EUA)-Baja California (México), En: Contaminación y Medio Ambiente en Baja California, Quintero M. Ed. Porrua, México, 3, 73-99 (2006).

NOM-020-SSA1-1993 Salud Ambiental., Criterio para evaluar la calidad el aire ambiente con respecto al ozono $\left(\mathrm{O}_{3}\right)$. Valor normado para la concentración de ozono $\left(\mathrm{O}_{3}\right)$ en el aire ambiente como medida de protección a la salud de la población, México (1993).

NOM-021-SSA1-1993 Salud ambiental., Criterio para evaluar la calidad del aire ambiente con respecto al monóxido de carbono (CO). Valor permisible para la concentración de monóxido de carbono (CO) en el aire ambiente como medida de protección a la salud de la población. México (1993). 
NOM-025-SSA1-1993. Salud ambiental., Criterios para evaluar el valor límite permisible para la concentración de material particulado. Valor límite permisible para la concentración de partículas suspendidas totales PST, partículas menores de 10 micrómetros $\mathrm{PM}_{10}$ y partículasmenores de 2.5 micrómetros $\mathrm{PM}_{2.5}$ de la calidad del aire ambiente. Criterios para evaluar la calidad del aire. México (1993).

Ocaña H. y otros cuatro autores; Contaminación atmosférica. Un enfoque global., Daños a la Salud por Contaminación atmosférica, Universidad Autónoma del Estado de México-IMSS, México, 244-261 (2001).

Omran A.; The Epidemiologic Transition, Milbank Memorial Fund Quarterly, 29, 509-538 (1971).

Pope C.; Air Pollution and Health-Good News and Bad, The New England Journal of Medicine: 11(351), 1132-1134 (2004).

Ramírez-Sánchez U., M. Andrade-García, M. González-Castañeda y A. Celis de la Rosa; Contaminantes atmosféricos y su correlación con infecciones agudas de las vías respiratorias en niños de Guadalajara, Jalisco, Salud Publica Mex.: 48(5), 385-394 (2006).

Reyna M., M. Quintero, K. Collins y L. Vildosola; Análisis de la relación del PM10 con las enfermedades respiratorias en la población urbana de Mexicali, Baja California: Un estudio de serie de tiempo, Revista Mexicana de Ingeniería Biomédica: XXIV(2), 116-125 (2003).

Rodríguez R. y N. Sánchez; Infecciones Respiratorias Agudas: aspectos clínicos y epidemiológicos: 5(7), 1-10 (2000).

Romero-Placeres M. y otros cinco autores: Contaminación atmosférica, asma bronquial e infecciones respiratorias agudas en menores de edad, de La Habana, Salud Publica Mex.: 46(3), 222-233 (2004).

Rosales-Castillo J., V. Torres-Mesa, G. Oláis-Fernández y V. Borja-Aburto; Los efectos agudos del aire en la salud de la población: evidencias de estudios epidemiológicos, Salud Publica Méx, 43(6), 544-555 (2001).

Samet J. y otros cuatro autores; Fine Particulate Air Pollution and Mortality in 20 U.S. Cities, 19871994, The New England Journal of Medicine: 24(343), 1742-1749 (2000).

San Martín H.; Salud y Enfermedad, La Prensa Medica Mexicana, S.A. de C.V. México, 5(II), 53-92 (1988).

SEDECO, Secretaria de Desarrollo Económico; Estadística Básica de Baja California (2005).

Smith K. y S. Akbar; Health-Damaging: A matter of Scale, Air Pollution and Health, in rapidly developing countries, Eathscan: 1(1), 21-34 (2003).

Sinclair H. y D. Tolsma; Associations old Lag between Air Pollution and Acute Respiratory Visits in an Ambulatory Care Setting: 25-Month Result from Aerosol Research and Inhalation Epidemiological Study, J. Air and Waste Managen. Assoc.: 54(9), 1212-1218 (2004).

SS, Secretaria de Salud, México; Morbilidad por Infecciones Respiratorias Agudas (2005) 